\title{
Hydrogel tip attached quartz tuning fork for shear force microscopy
}

\author{
Juhee $\mathrm{Ko}^{1}$, Amun Jarzembski ${ }^{2}$, Keunhan Park ${ }^{2}$ and Jungchul Lee ${ }^{3^{*}}$
}

\begin{abstract}
This paper reports the first demonstration of hydrogel conical tip attachment onto quartz tuning fork (QTF) by using an elastomeric tip mold that is soft-lithographically replicated from an electrochemically etched tungsten wire. The tungsten tip of 10-100 nm radius obtained by time-controlled electrochemical etching is replicated with h-polydimethylsiloxane (h-PDMS) to make negative conical tip molds large enough to be used for QTFs. By approaching a QTF to the negative h-PDMS tip mold filled with polyethylene glycol-diacrylate (PEGDA), a PEGDA tip is attached to the QTF without using an adhesive. Then, the PEGDA tip attached QTF is employed for shear force microscopy for calibration grating and atomic layers of hexagonal silicon carbide and also compared with a silicon tip attached QTF. Exclusively for the PEGDA tip attached QTF, we demonstrate that the imaging tip could be regenerated multiple times to address issues associated with tip wear. In a stark contrast with conventional QTF probes in attachment of electrochemically etched metallic wires or microfabricated AFM cantilevers, photocuring of liquid phase prepolymer within a tip mold demonstrated herein allows adhesive-free and exclusive attachment of the imaging tip onto a QTF. The relatively large PEGDA tip enables facile operation during approach and engagement. Moreover, the organic and inorganic combination of imaging tip and resonating body offers regeneration of the imaging tip upon its degradation.
\end{abstract}

Keywords: Atomic force microscopy (AFM), Hydrogel tip, Shear force, Tip attachment, Quartz tuning fork (QTF)

\section{Background}

Although atomic force microscopy (AFM) probes have been typically made of silicon that is the second most abundant atom on earth, disposal of the whole probes is neither cost-effective nor environment-friendly considering the fabrication cost/time and hazardous chemicals used during fabrication. Therefore, it would be ideal if the worn or damaged imaging tip could be independently replaced. The recent study revealed that a PEGDA tip shows superior wear-resistance to silicon tip due to the extended attractive regime for the PEGDA tip, which makes the true noncontact mode imaging more favorable [1]. In addition to the systematic wear comparison, it was shown that a worn or damaged PEGDA tip could be regenerated multiple times by chemically removing it and

\footnotetext{
*Correspondence: jungchullee@kaist.ac.kr

${ }^{3}$ Department of Mechanical Engineering, Korea Advanced Institute of Science and Technology, 291 Daehak-ro, Yuseong-gu, Daejeon 34141, Republic of Korea

Full list of author information is available at the end of the article
}

subsequently attaching a new PEGDA tip to a tipless silicon cantilever [2]. The multiple regeneration was guaranteed by the etch selectivity of silicon in piranha solution that exclusively removed the PEGDA tip.

However, tipless silicon cantilevers are still not costeffective to fabricate, and their resonance frequencies exhibit intrinsic uncertainty originating from non-uniform wafer thickness and spatiotemporal process variation during fabrication. On the other hand, QTFs typically used for timing references in wrist watches [3] are orders of magnitude cheaper than tipless silicon cantilevers and exhibit very uniform frequency characteristics and high quality factors. Sizes of commercial QTFs are much larger than that of tipless silicon cantilevers to guarantee much easier manufacturing and handling. In addition, the QTFs are more robust than tipless silicon cantilevers and tend to survive even after physical impact by accident. Due to the aforementioned benefits, it would be advantageous if the PEGDA tip can be attached and regenerated onto QTFs. While attaching a PEGDA tip to QTFs seems much easier than attaching 
a microfabricated silicon cantilever $[4,5]$ or an etched tungsten wire [6], the negative tip mold previously used for tipless silicon cantilevers [2] is too small for QTFs: it may prevent easy use of the probe for imaging after tip attachment. A simple and straightforward solution for this issue is to make a larger negative tip mold. However, it requires extended processing time as well as thicker wafers to make larger tip mold masters with silicon via potassium hydroxide $(\mathrm{KOH})$ etching.

This paper reports the fabrication of relatively large negative elastomeric tip molds replicated from electrochemically etched tungsten wires for the first time. Using the large negative tip molds, sufficiently large but sharp PEGDA tips are fabricated and attached to QTFs by single ultraviolet (UV) exposure without using an adhesive. PEGDA tip attached QTFs are successfully employed for imaging of calibration grating and silicon carbide monolayers. In addition, the used PEGDA tip is removed by piranha solution and a new PEGDA tip is attached to demonstrate the modular AFM concept where the imaging tip can be replaced and regenerated if necessary.

\section{Experimental details}

\section{Electrochemical etching of tungsten wires}

The first step for the negative tip mold fabrication is to make a sharp tungsten tip by time-controlled electrochemical etching of a tungsten wire. This process is traditionally used to make a sharp metallic tip for scanning tunneling microscopy [7]. A ring electrode with the diameter of $3.5 \mathrm{~cm}$ is used to generate axisymmetric current flow around the immersed tungsten wire thus helps the etched tungsten tip to have an axisymmetric conical shape. Since the tungsten tip becomes blunt if the current flow is prolonged after the end of the etching process, a high-speed voltage comparator is employed in the feedback circuit to timely shut off the bias voltage immediately after the voltage monitored goes below a pre-defined threshold [8]. Hence, we construct the cutoff circuit as shown in Fig. 1a. When the voltage suddenly becomes lower than the threshold, the immersed segment of the tungsten wire drops off by its weight and it causes sudden drop in voltage. When the comparator detects such event, the bias voltage is immediately powered off. Figure 1b, c show scanning electron micrographs (SEM) of electrochemically etched tungsten wires of which diameters are $250 \mu \mathrm{m}$ and resulting tip heights as a function of immersion depth and sodium hydroxide $(\mathrm{NaOH})$ concentration. In general, the overall tungsten tip height increases as the immersion depth or $\mathrm{NaOH}$ concentration increases. Since an excessive immersion depth turns out to adversely affect tip sharpness, the immersion depth was optimized to be $\sim 2 \mathrm{~mm}$ in an effort to compromise between experimental costs for shallow immersion and etched tip quality. On the other hand, the lower $\mathrm{NaOH}$ concentration increases the overall electrochemical etching time while the higher $\mathrm{NaOH}$ concentration tends to increase tip radius. The optimal $\mathrm{NaOH}$ concentration was experimentally determined to be $\sim 3 \mathrm{M}$ in our electrochemical etching setup. Therefore, the immersion depth of $2 \mathrm{~mm}$ and $\mathrm{NaOH}$ concentration of $3 \mathrm{M}$ are used hereafter. Figure $1 \mathrm{~d}$ shows the monitored voltage during the electrochemical etching process of a $250-\mu \mathrm{m}$ diameter tungsten wire. In the beginning, there is a voltage spike due to an input surge current. After $\sim 10 \mathrm{~s}$, the voltage for monitoring the electrochemical etching process is normally obtained. As tungsten etching progresses, the voltage decreases monotonically with a constant rate as the cross-sectional area of tungsten wire decreases. At around $380 \mathrm{~s}$, the comparator detects the monitored voltage lower than the threshold of $1.4 \mathrm{~V}$ thus the etching process is terminated.

\section{Tip mold fabrication and hydrogel tip attachment}

Figure 2a shows the step-by-step process of the conical h-PDMS tip mold [9] preparation and the PEGDA tip attachment to a QTF. (i) First, the etched tungsten tip is inserted into the drilled acrylic plate and the space between the drilled hole and the tip is covered with epoxy. (ii) The tungsten tip and acrylic plate assembly suppresses the meniscus of replicating h-PDMS not to exceed the size of the etched tip. Then, the tungsten tip-acrylic plate assembly along with $100 \mu \mathrm{L}$ of silane (Trichlorosilane 98\%, JSI Silicon) is placed in a vacuum chamber for $30 \mathrm{~min}$, taken out of the vacuum chamber, and heated for $2 \mathrm{~h}$ at $60{ }^{\circ} \mathrm{C}$. This heating process completes the formation of a silane monolayer [10] that helps demolding after the h-PDMS replication. (iii) Next, the silanized tungsten tip-acrylic plate is placed over h-PDMS prepolymer prepared by following a recipe in [11]. After heating $45 \mathrm{~min}$ at $90{ }^{\circ} \mathrm{C}$, the h-PDMS is fully cured and then the silanized tungsten tip-acrylic plate is demolded from the cured h-PDMS. (iv) Then, PEGDA is prepared by mixing the PEGDA monomer with $1 \mathrm{wt} \%$ of photo-initiator (phenylbis (2,4,6-trimethylbenzoyl) phosphine oxide) and dispensed over the negative h-PDMS tip mold by using a pipette cut with a razor blade to make a sharp beveled end. (v) Optionally, the PEGDA filled tip mold can be bi-axially compressed to enhance the tip sharpness as well as the tip aspect ratio. (vi) After the QTF makes a hard contact with the PEGDA filled molds, UV is exposed to cure the PEGDA.

Figure $2 \mathrm{~b}$ shows a $3 \mathrm{D}$ drawing of the tip attachment setup with the mold compression jig, where a CCD camera monitors the attachment process from below. With this setup and the process aforementioned, the tip can be exclusively attached to QTFs at a desired position 


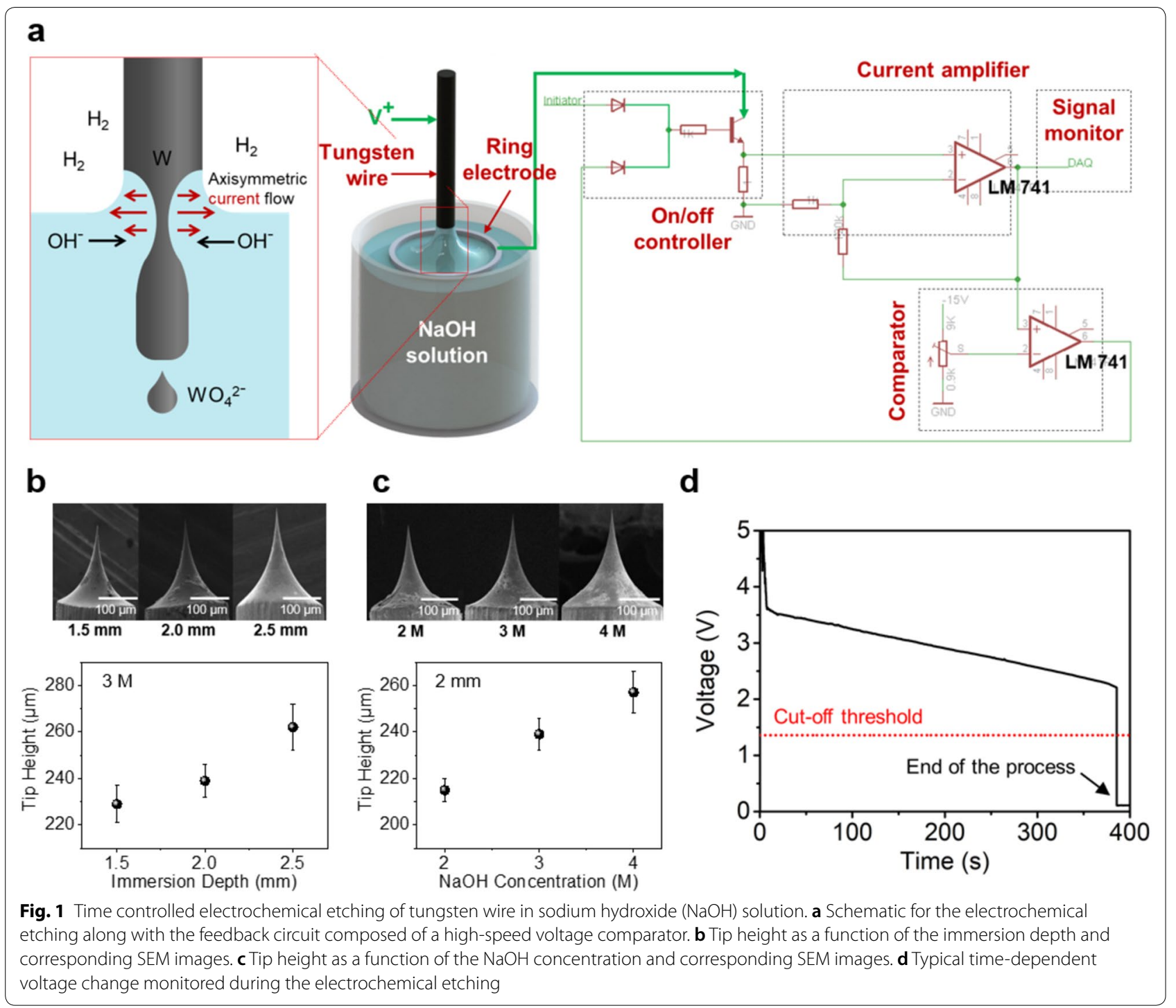

without using an adhesive. Figures $2 \mathrm{c}, \mathrm{d}$ compare a tungsten tip fabricated by electrochemical etching and a replicated PEGDA tip pair of which radii are 26 and $29 \mathrm{~nm}$, respectively. It should be noted that the sub- $30 \mathrm{~nm}$ radius PEGDA tip is the best result to date. However, such a high-quality PEGDA tip is not routinely obtained due to uncertainties in the tip mold fabrication and compression processes. The average PEGDA tip radius is around $250 \mathrm{~nm}$ with $10 \%$ variability. If necessary, plasma sharpening [12] can be used to further reduce the tip radius. The optional bi-axial compression may also be necessary to achieve a tip radius smaller than $50 \mathrm{~nm}$. The relationship between PDMS mold compression and PEGDA tip sharpness enhancement was studied in the previous study, reporting the improvement of a tip radius by 10 folds with the $15 \%$ increase of bi-axial compression [13].
Although the initial dimension of the tip mold and mold materials are different, the underlying physics is identical when applying the same strain, so we expect similar enhancement.

\section{Dynamic characterization}

While QTFs are electrically driven, QTF dynamics is characterized optically (Fig. 3a) and electrically (Fig. 3b) by implementing a commercial laser Doppler vibrometer equipped with a custom QTF drive and lock-in detection circuit [14]. Figure $3 \mathrm{a}, \mathrm{b}$ show the resonance spectrum change of a QTF before and after the PEGDA tip is attached. To precisely extract the resonance frequency and the quality factor, optically measured spectra are fitted with a simple harmonic oscillator model [15] and electrically measured are 


\section{a}

i Tip length adjustment ii Surface treatment

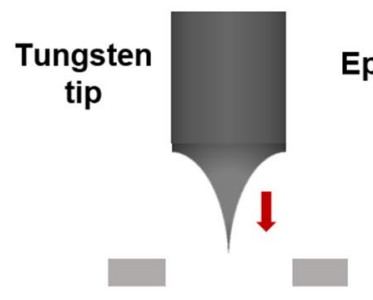

Drilled acrylic plate

iv Hydrogel dispensing
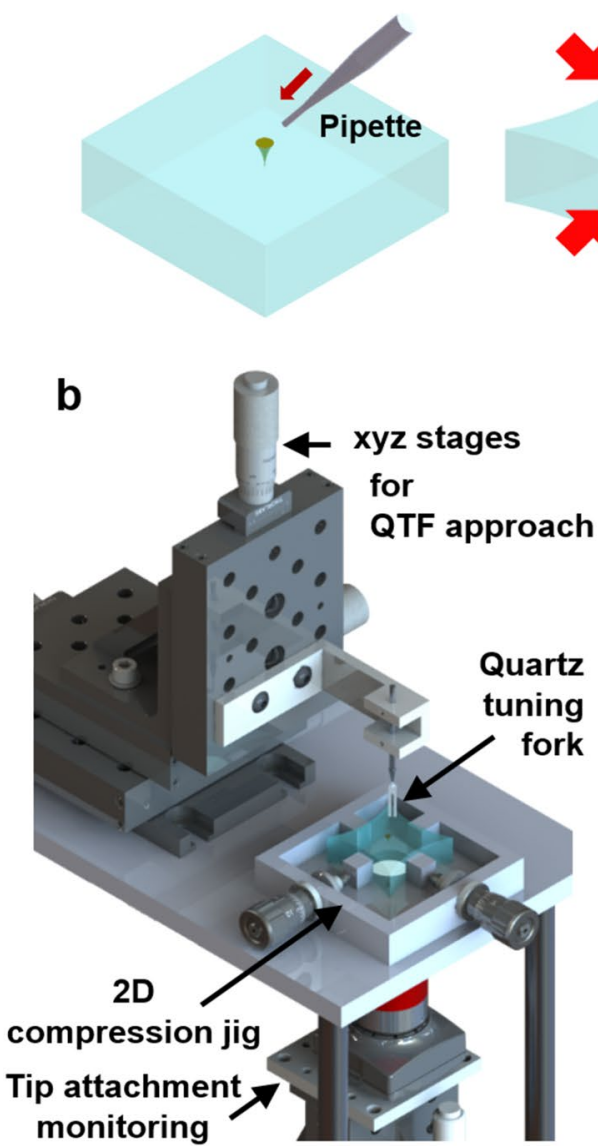

C

d iii Tip mold fabrication

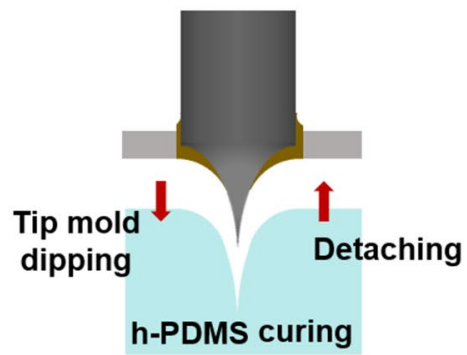

vi Tip attachment

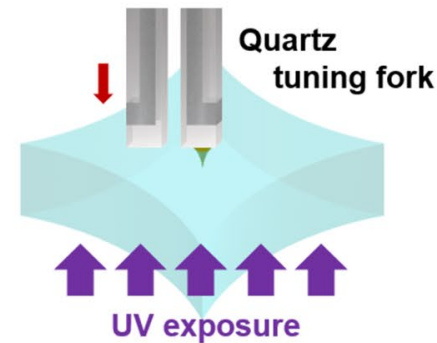

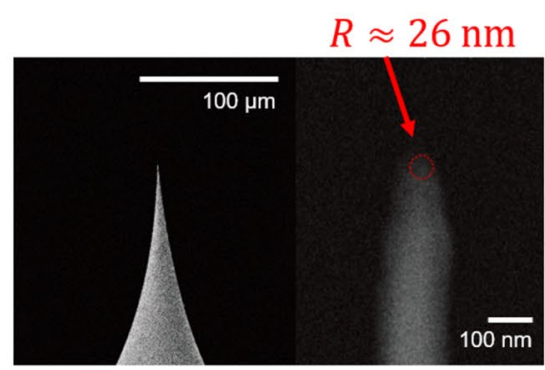

$R \approx 29 \mathrm{~nm}$

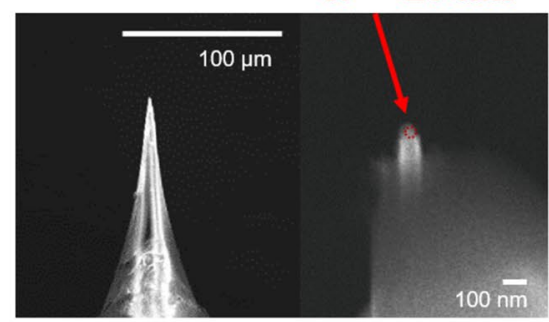

Fig. 2 Tip attachment process, setup, and results. a Process of making conical tip mold and attachment of hydrogel (PEGDA) tip onto quartz tuning fork. $\mathbf{b}$ 3D CAD drawing of the tip attachment setup with the mold compression jig. SEM images of $\mathbf{c}$ an electrochemically etched tungsten wire and $\mathbf{d}$ a PEGDA tip replicated from the etched tungsten wire and attached to quartz tuning fork

fitted with the Butterworth-van-dyke equivalent circuit model [16]. In our experiments, the in-plane anti-phase resonance of the QTFs is measured to be $\sim 32.768 \mathrm{kHz}$ after their package cans are manually removed. Considering the average volume $(0.2 \mathrm{~nL})$ of negative h-PDMS tip molds and density of PEGDA at $25^{\circ} \mathrm{C}(1.11 \mathrm{~g} / \mathrm{mL})$, the average mass of attached PEGDA tips is estimated to be $0.21 \mu \mathrm{g}$. Since the tip attachment induces the frequency shift of $\sim 120 \mathrm{~Hz}$, the mass responsivity is calculated to be $\sim 571.4 \mathrm{~Hz} / \mu \mathrm{g}$. The quality factor of the QTF is $\sim 4000$ as extracted from both optical and electrical measurements, which remains almost unchanged regardless of tip attachment. 


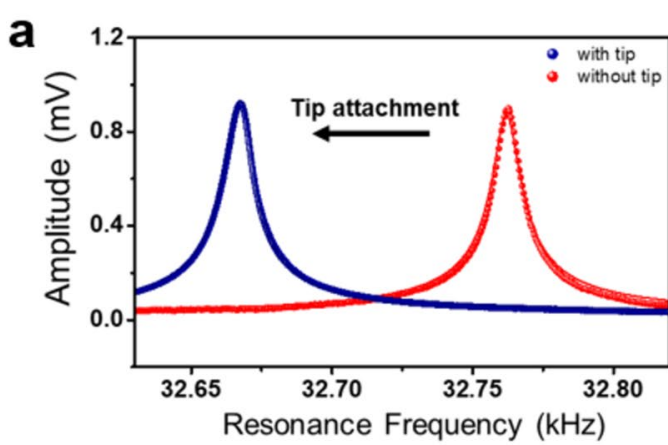

C

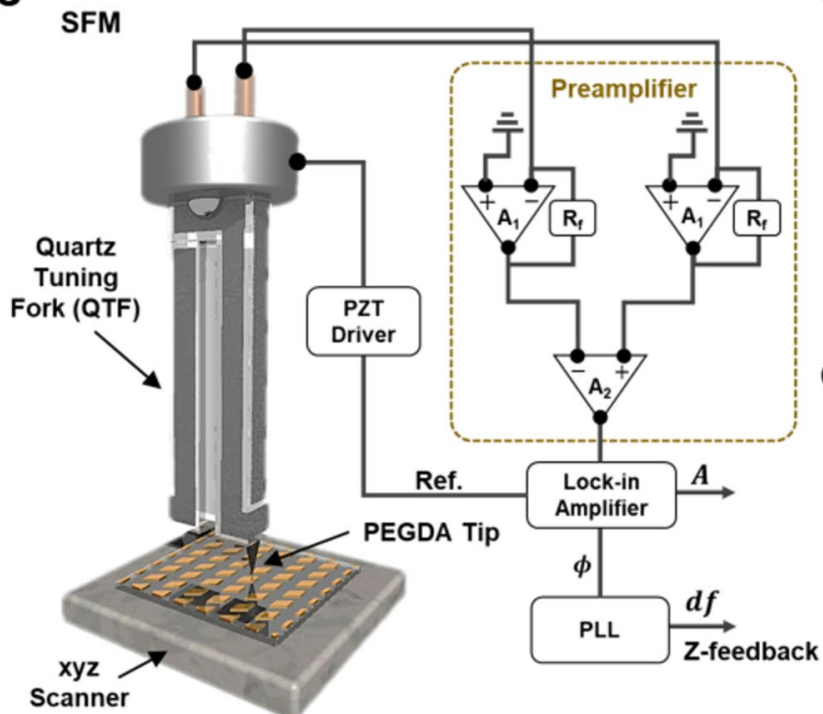

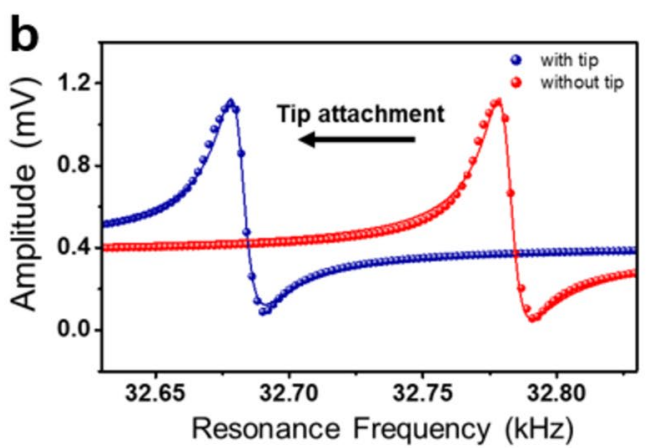

d
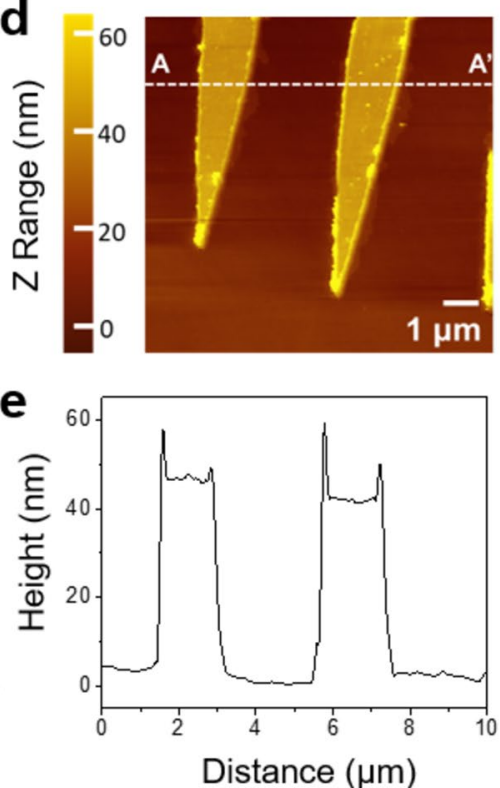

Fig. 3 Shear force microscopy (SFM) with the PEGDA tip attached quartz tuning fork. Amplitude spectra of QTFs before and after the PEGDA tip attachment by a optical and $\mathbf{b}$ electrical readouts. $\mathbf{c}$ Schematic and circuit diagram for SFM. $\mathbf{d}$ Dynamic mode topography and $\mathbf{e}$ a linescan of a calibration sample along the dashed line $A-A^{\prime}$

\section{Calibration grating imaging}

In shear force microscopy (SFM), the QTF is mechanically driven by a dithering piezo-actuator (PZT) at its in-plane anti-phase resonance, such that the tip's motion is predominantly lateral [17]. This enables shear (or frictional) force interaction of the tip with the sample surface. Under the shear force, the piezoelectric property of the QTF itself generates a current proportional to the deflection of the QTF prongs. Figure $3 \mathrm{c}$ represents operational schematic of the SFM, which utilizes a vertically aligned QTF with a PEGDA tip attached to the end of one of two prongs. Due to the high Q-factor of the QTF, the resonance frequency shift $(\Delta f)$ of the QTF probe is measured by a phase-locked loop (PLL) and used as the set point for the $\mathrm{z}$-feedback control of the sample stage. While a PEGDA tip scans over a sample surface, surface topography can be precisely measured by monitoring the vertical displacement of the sample stage to maintain $\Delta f$ at the set point. The QTF electrical signal is detected with two amplifiers ( $\mathrm{A}_{1}$ : AD8626, Analog Devices) with the trans-impedance gain $\left(R_{\mathrm{f}}\right)$ of $1 \times 10^{8}$ $\mathrm{V} / \mathrm{A}$, from which a differential signal is amplified $\left(\mathrm{A}_{2}\right.$ : AD8228, Analog Devices) with a gain of 10. Therefore, the output of the SFM pre-amplifier is a voltage signal proportional to the QTF oscillation amplitude. The signal from the SFM pre-amplifier is demodulated by using a lock-in amplifier at the dithering piezo drive frequency, yielding the amplitude $(A)$ and phase $(\Phi)$ of the QTF oscillation. After the resonance frequency and vibration amplitude are measured as a function of the tip-sample distance during approach and retract 
cycle, noncontact-mode shear force imaging is performed on the calibration sample $(\sim 50 \pm 10 \mathrm{~nm}$ thick vanadium rhombus array on a quartz substrate): see Fig. 3d. Figure 3e shows a line profile of the calibration sample along the white dashed line $\mathrm{A}-\mathrm{A}^{\prime}$ in Fig. 3d.

\section{Result and discussion}

Comparison of commercial silicon tip and PEGDA tip

After imaging the calibration sample, a silicon carbide monolayer $(6 \mathrm{H}-\mathrm{SiC})[18,19]$ is imaged with a PEGDA tip. In addition, the imaging performance of the PEGDA tip is compared with that of a silicon tip. For the silicon tip

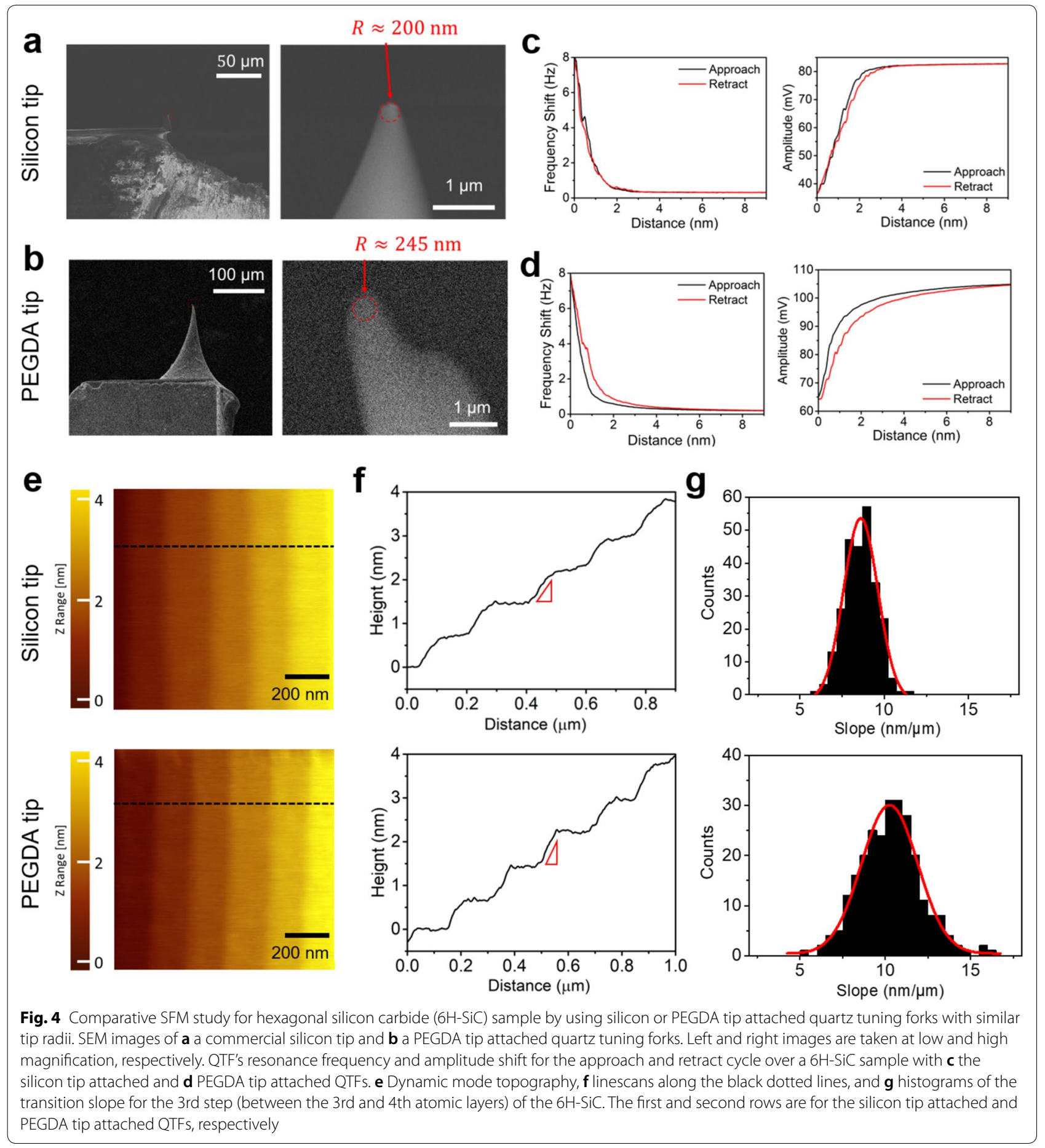


probe, a commercial AFM cantilever (Bruker, FMV-A) having a tip radius of $\sim 200 \mathrm{~nm}$ due to repeated uses was attached to a QTF using a small amount of UV curable epoxy (Bondic): see Fig. 4a. To make fair comparison, the PEGDA tip attached QTF of which radius is $\sim 245 \mathrm{~nm}$ was selected as shown in Fig. 4b. Figure 4c, d show the $z$-spectroscopy of the oscillation amplitude and the resonance frequency shift for the two QTF probes shown in Fig. 4a (silicon tip) and 4b (PEGDA tip), respectively, as the QTF approaches to and retracts from a $6 \mathrm{H}-\mathrm{SiC}$ monolayer sample. As the QTF probe approaches the sample, the oscillation amplitude decreases due to the increase of the damping while the QTF resonance frequency increases due to the increase of the stiffness associated with positive force gradient and repulsive tip-sample interaction. Therefore, the overall trends are similar for silicon tip attached and PEGDA tip attached QTFs. However, the PEGDA tip starts to interact with the underlying sample at larger tip-sample distance, as manifested by the changes of the oscillation amplitude and the resonance frequency of the QTF in Fig. 4d. Although the underlying physics of such an earlier recognition of the nearby substrate is not yet exactly known, the PEGDA tip attached QTF at least seems more appropriate for true noncontact mode imaging due to the wider attractive regime. Another observation is that the hysteresis between approach and retract cycle is more pronounced for the PEGDA tip attached QTF. However, it is not our main interest since the hysteresis plays no role during the SFM.

By setting a proper setpoint, topography of the $6 \mathrm{H}-\mathrm{SiC}$ monolayer can be obtained along with amplitude and phase errors. Figure $4 \mathrm{e}$ shows a topographic image of the $6 \mathrm{H}-\mathrm{SiC}$ monolayer sample where the setpoint, scan speed and scan area are $8 \mathrm{~Hz}, 0.5 \mu \mathrm{m} / \mathrm{s}$ and $1 \mu \mathrm{m} \times 1 \mu \mathrm{m}$, respectively. Quick side note here, shear force exerting on the tip during the imaging can be estimated to be $\sim 67 \mathrm{nN}$, which may be not sufficient to detach the tip from the QTF. During routine imaging with such a condition, we have not observed the detachment of hydrogel tips from QTFs.

Figure 4f shows line profiles extracted from the black dashed lines in Fig. 4e where each step is equal to half of the lattice constant of the silicon carbide (i.e., $7.5 \AA$ ). Figure $4 \mathrm{~g}$ represents histograms of the transition slope for the 3rd step between the 3rd and 4th atomic layers of the $6 \mathrm{H}-\mathrm{SiC}$ where all 256 linescan data are used. Each histogram is fitted to Gaussian to obtain average and standard deviation, yielding $8.57 \pm 0.91$ and $10.30 \pm 1.77 \mathrm{~nm} /$ $\mu \mathrm{m}$ for the silicon tip and the PEGDA tip, respectively. Although the PEGDA tip exhibits larger variation, its average slope is 1.2 times steeper than that for the silicon tip. The steeper transition with the PEGDA tip may be because the meniscus effect around the tip is more dominant for the silicon tip. In addition, the larger variation of the transition slope may be attributed to mechanical deformation of the relatively soft PEGDA tip. From a practical point of view, the silicon tip attached QTF is more challenging to handle than the PEGDA tip attached QTF mainly due to the relatively small size of the imaging tip. If a small tip attached QTF is slightly tilted during engagement to a sample surface, other corners of the QTF would touch the sample prior to the attached tip and damage the sample surface or produce image artifacts.

\section{Tip regeneration}

To address wear and damage of the attached PEGDA tip, the same tip regeneration scheme for tipless silicon cantilevers [2] is adopted for QTFs. The piranha solution prepared by mixing sulfuric acid (96\%) and hydrogen peroxide $(30 \%)$ with $3: 1$ volume ratio is used at room temperature to selectively remove the attached PEGDA tip presumably worn or damaged due to repeated uses. During the piranha process for about $20 \mathrm{~min}$, inorganic QTFs remain intact [20]. After the PEGDA tip is completely removed, QTF surfaces are rinsed and cleaned with DI water for $10 \mathrm{~min}$ and then dried. Next, a new PEGDA tip is attached. Figure 5a shows the SEM images of the first tip attachment, the first tip removal, and the second tip attachment onto QTF, respectively. The tip radii of the original and regenerated tips are 224 and $255 \mathrm{~nm}$, respectively. This regeneration process can be repeated multiple times. To demonstrate this, different PEGDA tips were attached to and removed from the same QTF three times, and the QTF resonant spectra were recorded in each case. Figure 5b, c show the amplitude spectra and resonance frequencies taken during the repeated regeneration, respectively. Red-shifts of the resonance frequency after the tip attachment are due to the added mass of the PEGDA tip [14]. The frequency variation of the QTF with PEGDA tip attached $(32,672 \pm 6 \mathrm{~Hz})$ is three times larger than that of the QTF with PEGDA tip removed $(32,777 \pm 2 \mathrm{~Hz})$. The relatively small frequency variation after tip removal implies that piranha cleaning selectively removes the PEGDA tip without affecting the QTF. In contrast, the relatively large variation after tip attachment is mostly attributed to the mass uncertainty of the attached PEGDA tip. The uncertainty of the attached tip mass is estimated to be $10.5 \mathrm{ng}$, which is about $5.7 \%$ of the average tip mass of $184 \mathrm{ng}$.

\section{Conclusions}

In this paper, we introduce an unconventional approach to fabricate hydrogel (PEGDA) tip attached QTFs for shear force atomic force microscopy. Using the h-PDMS tip mold replicated from an electrochemically etched 
a $1^{\text {st }}$ tip attach Tip remove $2^{\text {nd }}$ tip attach

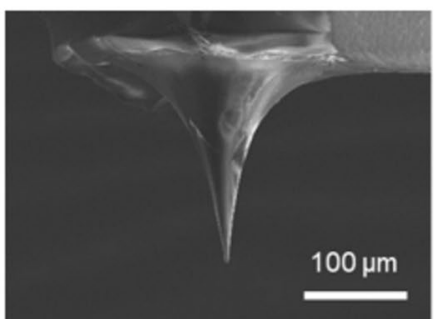

b

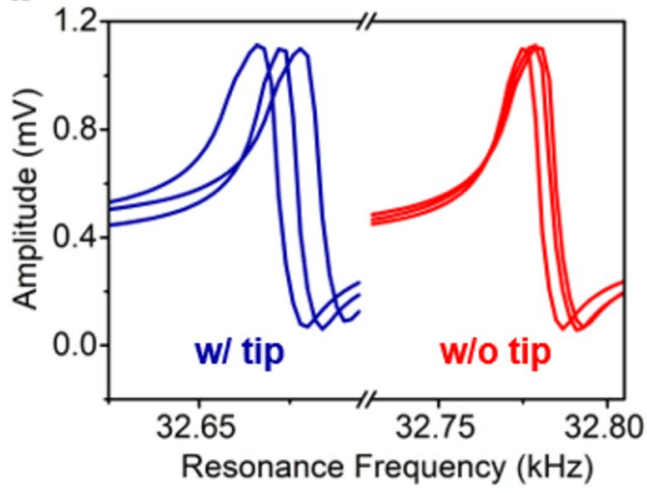

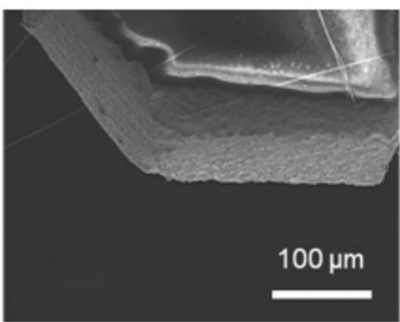

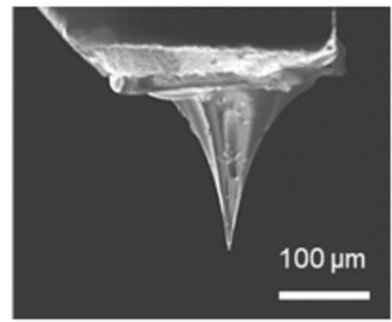

C

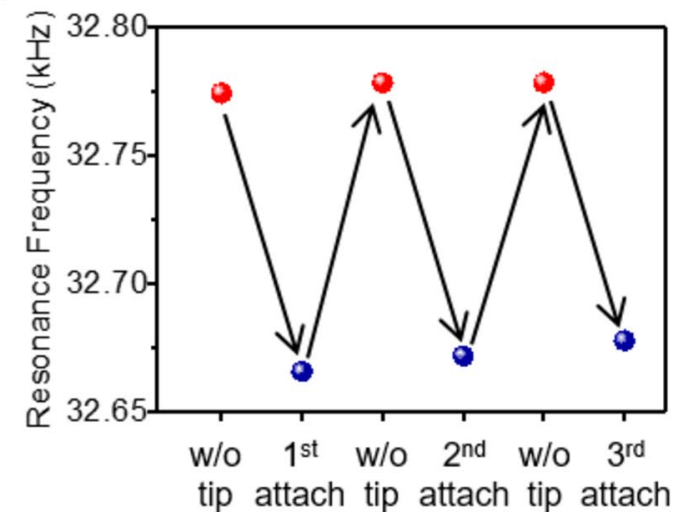

Fig. 5 Tip regeneration. a SEM images for the 1st tip attachment, 1st tip removal, and 2nd tip attachment. b Amplitude spectra taken after tip attachment and tip removal. c Resonance frequency of the QTF recorded during the tip regeneration of 3 cycles

tungsten wire, a PEGDA tip can be spontaneously attached to QTFs upon UV exposure without using an adhesive. PEGDA tip attached QTFs are implemented to image calibration grating and atomic layers of hexagonal silicon carbide samples in an SFM platform, demonstrating the advantages of the PEGDA tip in noncontact shear force imaging over a silicon tip. The proposed tip attachment method also offers repeated tip regeneration without damaging the QTF when tip wear or damage is not tolerable for imaging. We believe that other AFM applications beyond topographic imaging can be explored with the PEGDA tip attached QTFs in near future.

\section{Authors' contributions}

JK and JL developed the idea. JK and JL designed, fabricated, and characterized PEGDA tips and PEGDA tip attached QTF probes while AJ and KP conducted the SFM measurements and imaging processes using the developed probes. JK mainly analysed the results with AJ's assistance, and JK and JL wrote the manuscript. All authors read and approved the final manuscript.

\section{Author details}

${ }^{1}$ Department of Mechanical Engineering, Sogang University, Seoul 04107, Republic of Korea. ${ }^{2}$ Department of Mechanical Engineering, Utah University, Salt Lake City, UT 84112, USA. ${ }^{3}$ Department of Mechanical Engineering, Korea Advanced Institute of Science and Technology, 291 Daehak-ro, Yuseong-gu, Daejeon 34141, Republic of Korea.

\section{Competing interests}

The authors declare that they have no competing interests.

\section{Funding}

This research was supported by the National Research Foundation of Korea (NRF) funded by the Korea government (MSIP) (NRF-2017R1A2B3009610 and NRF-2017R1A4A1015564) was also supported by the National Science Foundation (CBET-1605584) and the University of Utah Funding Incentive Seed Grant. AJ also acknowledges financial supports from the University of Utah's Sid Green Fellowship and the National Science Foundation Graduate Research Fellowship (No. 2016213209).

\section{Publisher's Note}

Springer Nature remains neutral with regard to jurisdictional claims in published maps and institutional affiliations.

Received: 30 August 2018 Accepted: 19 November 2018 Published online: 22 November 2018

\section{References}

1. Kim S, Yoon Y, Lee J (2017) Direct assembly of a hydrogel nano-tip onto silicon microcantilevers for wear study and facile regeneration of soft atomic force microscope probes. Proc MEMS 2017:16708111

2. Lee J, Kim D, Yoon Y, Lee B, Ko J, Lee J (2018) Hydrogel tip integration onto tipless silicon cantilevers for atomic force microscopy and its facile regeneration. JMEMS Lett. 7:125-126

3. Friedt JM, Carry E (2007) Introduction to the quartz tuning fork. Am J Phys 75:415-422

4. Wu Z, Guo T, Tao R, Liu L, Chen J, Fu X, Hu X (2015) A unique selfsensing, self-actuating AFM probe at higher eigenmodes. Sensors 15:28764-28771 
5. Akiyama T, Staufer U (2003) Symmetrically arranged quartz tuning fork with soft cantilever for intermittent contact mode atomic force microscopy. Rev Sci Instrum 74:112-117

6. Gao F, Li X (2015) Research on the sensing performance of the tuning fork-probe as a micro interaction sensor. Sensors 15:24530-24552

7. Milhim AB, Mrad RB (2014) Electrochemical etching technique: conicallong-sharp tungsten tips for nanoapplications. J Vac Sci Technol B 32:031806

8. Khan Y, Al-Falih H, Zhang Y, Ng TK, Ooi BS (2012) Two-step controllable electrochemical etching of tungsten scanning probe microscopy tips. Rev Sci Instrum 83:063708

9. Schmid H, Michel B (2000) Siloxane polymers for high-resolution, high accuracy soft lithography. Macromolecules 33:3042-3049

10. Le Grange JD, Markham JL (1993) Effects of surface hydration on the deposition of silane monolayers on silica. Langmuir 9:1749-1753

11. Li H, Beinn V, Muir O, Fichet G, Wilhelm T, Huck S (2003) Nanocontact printing: a route to sub-50-nm-scale chemical and biological patterning. Langmuir 19:1963-1965

12. Jung B, Kong H, Cho Y, Park C, Kim M, Jeon B, Yang D, Lee K (2013) Fabrication of $15 \mathrm{~nm}$ curvature radius polymer tip probe on an optical fiber via two-photon polymerization and $\mathrm{O}_{2}$-plasma ashing. Curr Appl Phys 13:2064-2069
13. Lee J, Song J, Kim S, Kim S, Lee W, Jackman JA, Kim D, Cho N, Lee J (2016) Multifunctional hydrogel nano-probes for atomic force microscopy. Nat Commun 7:11556

14. Ko J, Yoon Y, Lee J (2018) Quartz tuning forks with hydrogel patterned by dynamic mask lithography for humidity sensing. Sensors Actuators B Chem 273:821-825

15. Lee I, Lee J (2013) Measurement uncertainties in resonant characteristics of MEMS resonators. J Mech Sci Technol 27:491-500

16. Ullah N, Park S, Lee Y (2015) Investigation of the electrical model parameters of quartz tuning forks from a low-frequency impedance analysis using a lock-in amplifier. New J Phys 65:76-80

17. Gomez AC, Agrait N, Bollinger GR (2009) Dynamics of quartz tuning fork force sensors used in scanning probe microscopy. Nanotechnology 20:215502

18. Yazdi GR, lakimov T, Yakimova R (2016) Epitaxial graphene on SiC: a review of growth and characterization. Crystals 6:53

19. Nagahara LA, Hashimoto K, Fujishima A, Snowdenlfft D, Price PB (1994) Mica etch pits as a height calibration source for atomic force microscopy. J Vac Sci Technol B 12:1694-1697

20. Kern W (1990) The evolution of silicon wafer cleaning technology. J Electrochem Soc 137:1887-1892

\section{Submit your manuscript to a SpringerOpen ${ }^{\circ}$ journal and benefit from:}

- Convenient online submission

- Rigorous peer review

- Open access: articles freely available online

- High visibility within the field

- Retaining the copyright to your article

Submit your next manuscript at $\boldsymbol{\nabla}$ springeropen.com 